\title{
Verifying competitive equilibria in dynamic economies
}

Kubler, Felix

\begin{abstract}
In this paper, I examine -equilibria of stationary dynamic economies with heterogeneous agents and possibly incomplete financial markets. I give a simple example to show that even for arbitrarily small $>0$, allocation and prices can be far away from exact equilibrium allocations and prices. That is, errors in market clearing or individuals' optimality conditions do not provide enough information to assess the quality of an approximation. I derive a sufficient condition for an -equilibrium to be close to an exact equilibrium. If the economic fundamentals are semi-algebraic, one can verify computationally whether this condition holds. The condition can be interpreted economically as a robustness requirement on the set of -equilibria which form a neighbourhood of the computed approximation. I illustrate the main result and the computational method using an infinite horizon economy with overlapping generations and incomplete financial markets.
\end{abstract}

DOI: https://doi.org/10.1093/restud/rdr005

Posted at the Zurich Open Repository and Archive, University of Zurich

ZORA URL: https://doi.org/10.5167/uzh-50759

Journal Article

Accepted Version

Originally published at:

Kubler, Felix (2011). Verifying competitive equilibria in dynamic economies. Review of Economic Studies, 78(4):1379-1399.

DOI: https://doi.org/10.1093/restud/rdr005 


\title{
Verifying Competitive Equilibria in Dynamic Economies*
}

\author{
Felix Kubler \\ ISB, University of Zurich \\ and Swiss Finance Institute \\ fkubler@gmail.com
}

November 28, 2010

\begin{abstract}
In this paper I examine $\epsilon$-equilibria of stationary dynamic economies with heterogeneous agents and possibly incomplete financial markets. I give a simple example to show that even for arbitrarily small $\epsilon>0$, allocation and prices can be far away from exact equilibrium allocations and prices. That is, errors in market clearing or individuals' optimality conditions do not provide enough information to assess the quality of an approximation. I derive a sufficient condition for an $\epsilon$-equilibrium to be close to an exact equilibrium. If the economic fundamentals are semi-algebraic, one can verify computationally whether this condition holds. The condition can be interpreted economically as a robustness requirement on the set of $\epsilon$-equilibria which form a neighborhood of the computed approximation.

I illustrate the main result and the computational method using an infinite horizon economy with overlapping generations and incomplete financial markets.
\end{abstract}

Keywords: Dynamic general equilibrium, semi-algebraic economy, computational methods

JEL Classifications: C63 D52 G12

\footnotetext{
*I thank seminar participants at various universities and conferences and in particular Alexandre Belloni, John Geanakoplos, Ken Judd, Ramon Marimon, Jean-Francois Mertens, Hamid Sabourian, Larry Samuelson and two anonymous referees for helpful comments.
} 


\section{Introduction}

Given a numerically computed $\epsilon$-equilibrium of a dynamic stochastic economy with heterogeneous agents, how can one verify whether or not this is a good approximation to an exact competitive equilibrium? In this paper, I show that if economic fundamentals are semi-algebraic, i.e. per period utility- and production-functions can be described by finitely many polynomials (see e.g. Blume and Zame (1993) or Kubler and Schmedders (2010a)), one can validate numerically when a computed $\epsilon$-equilibrium provides a good approximation to an exact equilibrium. The basic idea is as follows. One can create a set of $\epsilon$-equilibria by constructing a neighborhood around the computed approximation. Under some regularity conditions explained in detail below, one can ensure that this set contains an exact equilibrium by verifying that, if the next period's endogenous variables lie in the set, the conditions necessary for competitive equilibrium imply that endogenous variables in the current period must also lie in the set. I show that this verification can be done relatively efficiently by using methods from polynomial optimization and therefore that the method can be used for medium-sized dynamic stochastic models. While this is only a sufficient condition for the computed approximation to be close to an exact equilibrium, I argue that, under an economically intuitive robustness requirement on the exact equilibrium, it is also necessary.

Applied researchers routinely compute $\epsilon$-equilibria of dynamic stochastic economies although almost nothing is known about the nature of exact equilibria in these models. For dynamic models where the solution can be characterized as a planner's problem, Santos and Vigo-Aguiar (1998) and Santos (2000) have developed sufficient conditions under which they can give explicit error bounds both on policy functions and on allocations. Under these conditions, error bounds on allocations can be derived from Euler equation residuals and $\epsilon$-equilibria are always close to exact equilibria. Unfortunately, however, these results do not generalize to models with heterogeneous agents and incomplete markets or overlapping generations. In these models, it is not known if recursive equilibria exist or if policies are continuous functions of the state (see e.g. Kubler and Polemarchakis (2004)). Even if, for given prices, each agent makes only a small error in his utility maximization problem, it is possible that the exact market clearing price is far from the computed approximation. The set of $\epsilon$-equilibria in these economies might therefore be very large and the computed approximation might be nowhere near to an exact equilibrium. In Section 2, I construct a simple example of a deterministic economy with overlapping generations where I find two approximate solutions. I can show that one $\epsilon$-equilibrium is close to an exact equilibrium while the other $\epsilon$-equilibrium, which exhibits similar errors in market-clearing and optimality conditions, is far away from any exact solution.

So far, no sufficient conditions were known which allow the derivation of error bounds on computed equilibrium prices and allocations in the models considered in this paper. Kubler and Schmedders (2005) show that, in these models, $\epsilon$-equilibria can be interpreted 
as exact equilibria of close-by economies. Their paper does not make any statements about the set of all $\epsilon$-equilibria or about how $\epsilon$-equilibria are related to exact equilibria of the given stationary economy ${ }^{1}$. However, it suggests that if a competitive equilibrium is wellbehaved (in a sense to be made precise later) perturbations of exogenous variables should not lead to large perturbations in the equilibrium and, if one considers the set of $\epsilon$-equilibria that results from such perturbations, it should form a well-behaved neighborhood around the exact equilibrium. In this paper, I build on this idea, derive a simple condition that ensures that a set of $\epsilon$-equilibria contains an exact equilibrium and argue that, in wellbehaved cases, a neighborhood of a computed $\epsilon$-equilibrium should satisfy this condition if it contains an exact equilibrium.

I define a set of $\epsilon$-equilibria to be robust if it satisfies the following property. Suppose at date $T$ economic fundamentals are perturbed so that from $T$ onwards the new competitive equilibrium realizes in the $\epsilon$-equilibrium set. Then robustness of this set requires that up to date $T$ the endogenous variables of the competitive equilibrium in which all agents anticipate the changes at $T$ also realize in the set. The main reason for introducing this concept is that robustness ensures that an $\epsilon$-equilibrium set contains an exact equilibrium and that one can effectively check if a (semi-algebraic) $\epsilon$-equilibrium set is robust. For semialgebraic economies, robust $\epsilon$-equilibrium sets that are semi-algebraic exist for all $\epsilon>0$. It is economically sensible to focus on competitive equilibria whose neighborhoods form robust $\epsilon$-equilibrium sets since otherwise small perturbations of fundamentals will lead to large changes in the equilibrium.

While the theoretical analysis is conducted using abstract equilibrium sets, one has to consider recursive $\epsilon$-equilibria for the practical error analysis. Computational algorithms typically use recursive methods to approximate equilibria numerically and for this $\epsilon$-equilibria are written as functions mapping the state of the economy into current endogenous variables. If this is the case, one of course wants to verify that the computed recursive $\epsilon$-equilibrium is close to an exact equilibrium for all permissable values of the state. In order to do so, I construct candidate robust $\epsilon$-equilibrium sets by creating a strip around a computed recursive $\epsilon$-equilibrium. I show that one can verify that the $\epsilon$-equilibrium set is robust for all relevant initial values of the state by solving a polynomial optimization problem. While this is not a convex programming problem, there now exist algorithms which find lower bounds on global minima of relatively large constrained polynomial problems (see Laurent (2008) for an overview).

Throughout the paper, I take as given that a candidate approximate equilibrium has been computed by some existing method and that this is described by a continuous and semialgebraic (policy-) function $\hat{\rho}$. While the existence of a recursive exact equilibrium is not guaranteed in general (Citanna and Siconolfi (2010) give conditions for the generic existence of recursive equilibria in economies with overlapping generations), recursive $\epsilon$-equilibria

\footnotetext{
${ }^{1}$ I illustrate the differences between the two approaches in the example in Section 2 .
} 
always exist. However, typically there is no guarantee that a recursive $\epsilon$-equilibrium can be described by continuous functions. This paper is not about the computation of $\epsilon$-equilibria (see Judd (1998) for detailed descriptions of algorithms for the computation of equilibria in dynamic stochastic models) but about the verification that a recursive $\epsilon$-equilibrium is close to an exact equilibrium. Even if there is a recursive $\epsilon$-equilibrium with a continuous policyfunction, it is not guaranteed that the construction of a candidate robust $\epsilon$-equilibrium set always works. However, if this is not the case, the economy is likely to be so 'badly-behaved' that it seems hopeless to derive accurate numerical solutions at all.

As an example, I study a stochastic economy with overlapping generations which is a generalization of Samuelson (1958) to uncertainty. In many applications, researchers routinely compute approximate equilibria for versions of this model and find that low degree polynomials suffice for very good approximations of policy-functions. This might strike one as surprising since it is well known that in many dynamic economic models with overlapping generations the set of competitive equilibria can be almost arbitrarily wild. In particular the issue of indeterminacy of equilibria in deterministic olg models received a lot of attention and it is now well understood that extremely restrictive assumptions are needed to guarantee local uniqueness (see e.g. Kehoe and Levine (1990)). But this obviously says little about the existence (or non-existence) of simple equilibria that can be approximated by lowdegree polynomials or by piece-wise polynomials. As Kehoe and Levine (1990) point out, in deterministic olg models the fact that the equilibrium set is complicated does not necessarily imply anything about the practical computation of equilibria.

The rest of the paper is organized as follows. In Section 2, I give a simple example to illustrate the main points of the paper. In Section 3, I abstractly describe the economy, define robust $\epsilon$-equilibrium sets and prove the main theoretical result that relates robust $\epsilon$-equilibria to exact competitive equilibrium. Section 4 introduces recursive methods and

relates the theoretical result to polynomial optimization. I argue in this section that whether or not a recursive $\epsilon$-equilibrium is close to an exact equilibrium can be verified by solving a series of constrained optimization problems. Section 5 applies the methods to examine simple dynamic equilibria in stochastic models with overlapping generations.

\section{An example}

To illustrate the main ideas, I examine one of the simplest examples where serious problems can arise. Consider an exchange economy with a single perishable commodity and overlapping generations. Time extends from zero to infinity, $t=0,1, \ldots$ At each $t$, a representative agent is born and lives for three periods. In each period, individuals receive endowments depending on their age, $e_{a}$ being the endowment of an agent of age $a=1, \ldots, 3$. Utility is 
time separable with the utility of an agent born at time $t$ given by

$$
U_{t}\left(c_{1}, c_{2}, c_{3}\right)=\sum_{a=1}^{3} \beta^{a} \frac{c_{a}^{1-\sigma}}{1-\sigma} .
$$

At each $t$, agents can trade in a risk-free bond with price $q(t)$. Let $\theta_{a}(t)$ denote the bondholding of an agent of age $a$ at time t. At $t=0$, the initial conditions of the economy are determined by the bond-holding of the initially alive agents of ages $a=1,2$.

A competitive equilibrium is defined as usual by market clearing and agent optimality, that is, it is given by a sequence $\left(q(t),\left(c_{a}(t)\right)_{a=1}^{3},\left(\theta_{a}(t)\right)_{a=1}^{2}\right)$ such that for each $t$, $\sum_{a=1}^{2} \theta_{a}(t)=0$ and such that each agent maximizes his utility given prices $(q(t))_{t=0}^{\infty}$.

Since utility is concave and satisfies an Inada condition, and agents are finitely lived, the first order conditions for agents' optimality are necessary and sufficient. A competitive equilibrium can therefore be described by first order conditions and market clearing. It is useful to define $z(t)$ to be the vector of all endogenous variables relevant at time $t$, i.e. $z(t)=\left(\theta_{1}(t-1), q(t),\left(c_{a}(t)\right)_{a=1}^{3},\left(\theta_{a}(t)\right)_{a=1}^{2}\right)$. Given initial conditions $\theta_{1}(-1)$, a competitive equilibrium can then be characterized as a sequence $(z(t))_{t=0}^{\infty}$ with $c_{a}(t) \geq 0$ for all $t$ and all $a=1,2,3$ that satisfies $h(z(t), z(t+1))=0$ for all $t=0,1, \ldots$, where

$$
h(z(t), z(t+1))=\left\{\begin{array}{l}
-q(t) \frac{1}{c_{1}(t)^{\sigma}}+\frac{\beta}{c_{2}(t+1)^{\sigma}} \\
-q(t) \frac{1}{c_{2}(t)^{\sigma}}+\frac{\beta}{c_{3}(t+1)^{\sigma}} \\
c_{1}(t)-e_{1}+q(t) \theta_{1}(t) \\
c_{2}(t)-e_{2}-\theta_{1}(t-1)+q(t) \theta_{2}(t) \\
c_{3}(t)-e_{3}+\theta_{1}(t-1) \\
\theta_{1}(t)+\theta_{2}(t)
\end{array}\right.
$$

The beginning-of-period wealth of the middle aged at time $t$ is given by $\theta_{1}(t-1)$, i.e. the savings of the young in the last period. It is convenient to build market-clearing into the definition of $z$ and $h$ and take the beginning of period wealth of the old to be $-\theta_{1}(t-1)$.

A competitive equilibrium is a steady state if there is a $\bar{z}$ such that $z_{t}=\bar{z}$ for all $t$, i.e. if there is a $\bar{z}$ with $h(\bar{z}, \bar{z})=0$. At least one steady state always exists in this example (the situation will be quite different once uncertainty is introduced). Generally, however, one is interested in competitive equilibria for initial conditions which are not part of a steady state. Ideally, one would like to describe competitive equilibria for an entire interval of initial conditions. In this case, one needs a convenient method to numerically describe or approximate the equilibrium. In this paper, I assume throughout that the approximate solution is in the form of a recursive $\epsilon$-equilibrium. In this example, the natural state space $\Theta$ consists of beginning-of-period bond-holdings of the middle aged and there are approximate policy-functions that map the state, $\theta_{-} \in \Theta$ into current period consumptions, savings and prices, $\hat{\rho}: \Theta \rightarrow \mathbb{R}_{+} \times \mathbb{R}_{+}^{3} \times \mathbb{R}^{2}$. That is for each $t, z(t)$ can be written as

$$
z(t)=\left(\theta_{1}(t-1), q(t),\left(c_{a}(t)\right)_{a=1}^{3},\left(\theta_{a}(t)\right)_{a=1}^{2}\right)=\left(\theta_{1}(t-1), \hat{\rho}\left(\theta_{1}(t-1)\right)\right.
$$


and satisfies for each $t,\left\|h\left(z_{t}, z_{t+1}\right)\right\|<\epsilon$.

For the concrete example, suppose there is no discounting $(\beta=1)$, that the coefficient of relative risk aversion is given by $\sigma=3$ and that individual endowments are $e_{1}=1, e_{2}=$ 10.575 and $e_{3}=0.5$. Suppose one is interested in equilibria for initial conditions $\theta_{-}=-6$.

I turns out that for this specification there exist recursive $\epsilon$-equilibria for which $\hat{\rho}$ can be written as a polynomial of degree 4 . It is not clear if polynomials are always the right choice in these economies as policies could be backward bending - however, for this example (and all the ones consider in Section 5 below) it turns out fine. There are the following two candidate solutions.

(1) The admissible state space is $\Theta=[-6,0]$ and approximate savings policy of the middle aged is given by

$$
\hat{\rho}_{\theta^{2}}\left(\theta_{-}\right)=0.000433 \theta_{-}^{4}+0.00224 \theta_{-}^{3}-0.000585 \theta_{-}^{2}+0.0289 \theta_{-}-0.154
$$

The bond price is given by

$$
\hat{\rho}_{q}\left(\theta_{-}\right)=-0.0012 \theta_{-}^{4}+0.0382 \theta_{-}^{3}+1.35 \theta_{-}^{2}+14.9 \theta_{-}+52.7
$$

(2) The admissible state space is $\Theta=[-6,-5.4]$ and the approximate savings policy (of the middle aged) is given by the following polynomial

$$
\hat{\rho}_{\theta^{2}}\left(\theta_{-}\right)=0.245 \theta_{-}^{3}+4.54 \theta_{-}^{2}+28.7 \theta_{-}+55.4 \text {. }
$$

The bond price is given by

$$
\hat{\rho}_{q}\left(\theta_{-}\right)=0.0385 \theta_{-}^{3}+0.717 \theta_{-}^{2}+4.56 \theta_{-}+10 .
$$

Figure 1 shows the approximate portfolio-policies for the two candidate solutions. Clearly the two equilibria are quite different. Solution (1) is inefficient, with very low consumption of the old, while Solution (2) is efficient with high consumption of the old.

In this simple example, one can verify that there is something 'wrong' with the second approximate solution as the model has a unique steady state at around $\theta_{-}=-0.15839$. The second solution seems to converge to an approximate steady state at around $\theta_{-}=-5.44571$, but all exact steady states in this model are characterized by a finite number of polynomial equations and the methods in Kubler and Schmedders (2010b) can be used to show that there is a unique steady state in this economy. In fact, building on this, I show in Appendix A of the paper that there cannot be a competitive equilibrium anywhere close to candidate solution (2), i.e. this $\epsilon$-equilibrium cannot be part of a cyclical equilibrium either.

The question is how to determine which one of these candidate solutions provides a good approximation to an exact equilibrium without knowing the steady states. As mentioned above, in models with stochastic shocks, there are no steady-state equilibria and the quality of an approximation has to be judged differently. The standard measure is relative errors 


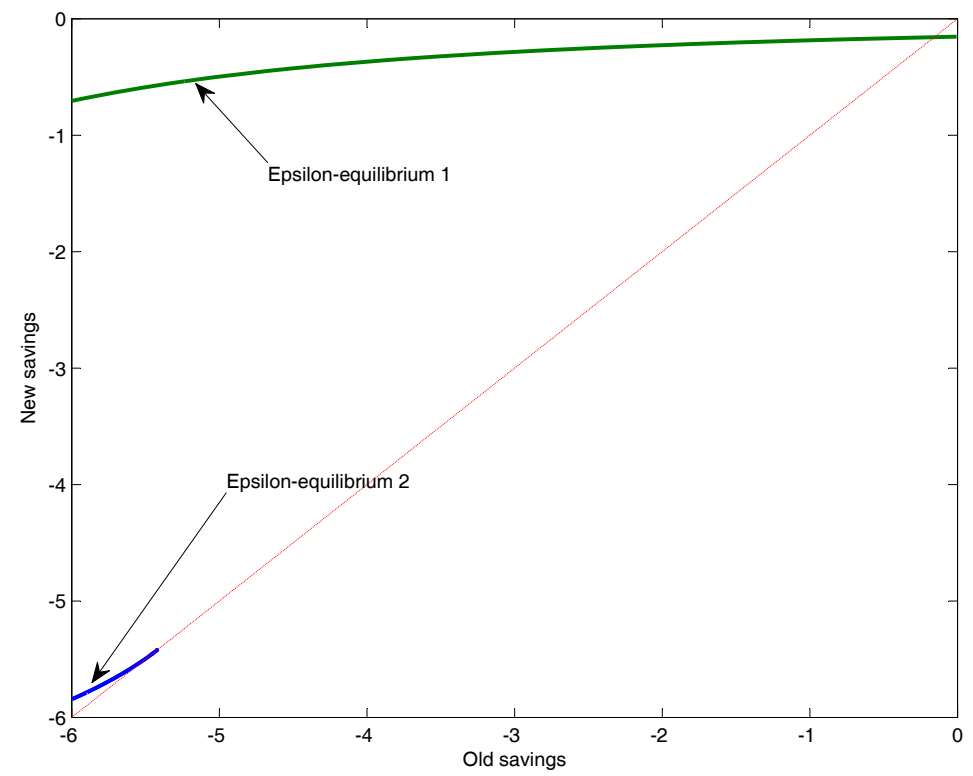

Figure 1

in Euler equations. That is, one can impose market clearing and budget-constraints on the approximate equilibrium by computing consumptions from the budget constraints. The only error is then in the Euler equations and it is useful (see Judd (1998)) to report the maximum relative error

$$
\epsilon^{r}=\max _{t}\left\{\max \left(\beta \frac{c_{2}(t+1)^{-\sigma}}{q(t) c_{1}(t)^{-\sigma}}-1, \frac{\beta c_{3}(t+1)^{-\sigma}}{q(t) c_{2}(t)^{-\sigma}}-1\right)\right\} .
$$

In both cases, these errors turn out to be very small (in solution 2 the maximum error is $10^{-5}$, in solution 1 around $2 \times 10^{-5}$ ) and therefore this criterion cannot discriminate between the good approximation and the candidate solution that is far from any exact equilibrium. In fact, it is clear from Santos (2000) that one can only infer from these errors how far away agents choices are from optimal choices, given fixed prices. In this example, both choices and prices are approximate.

The analysis in Kubler and Schmedders (2005) implies that for both approximate equilibria one can construct some 'close-by' economy so that these equilibria become exact. In fact, it turns out that for a slightly larger endowment of the middle-aged, e.g. for $e^{2}=10.576$, the economy has three steady states and the second candidate solution is close 
to an exact solution. However, for $e^{2} \leq 10.575$, there is certainly no exact equilibrium anywhere close-by. Therefore, it is not possible to conduct local comparative statics in any meaningful way and the second candidate solution is not robust even as an $\epsilon$-equilibrium. In a stochastic setting the situation is even worse. The construction in Kubler and Schmedders (2005) generally does not yield a stationary economy and it is possible that there is no 'close-by' economy that is stationary and for which the computed equilibrium is exact. Even if one performs careful robustness analysis and computes many examples with similar endowments and preferences, it is possible that one never obtains $\epsilon$-equilibria that are good approximations of exact competitive equilibria for the stationary economies under consideration.

In the following two sections, I will describe a method which can be used to prove that the first candidate solution is close to an exact equilibrium. In Section 5, I will illustrate the method using the example from this section.

\section{$3 \quad$ An abstract model}

In order to formally present the main theoretical result, I first introduce a general stochastic dynamic framework that fits both models with overlapping generations and models with infinitely lived agents and incomplete markets. At this stage, I do not assume that the approximate equilibrium is recursive, since the main result is easiest to prove in a general framework.

\subsection{The Dynamic Economy}

I consider a general abstract formulation of dynamic general equilibrium. Duffie et al. (1994) use a similar framework (not assuming differentiability and semi-algebraic fundamentals, as I do) and show in their paper that it encompasses general equilibrium models with overlapping generations as well as models with infinitely lived agents. It will turn out that most dynamic general equilibrium models used in applications fit the framework.

Time and uncertainty are represented by a countably infinite tree $\Sigma$. Each node of the tree, $\sigma \in \Sigma$, is a finite history of shocks $\sigma=s^{t}=\left(s_{0}, s_{1}, \ldots, s_{t}\right)$ for a given initial shock $s_{0}$. The process of shocks $\left(s_{t}\right)$ is assumed to be a Markov chain with finite support $\mathcal{S}$. To indicate that $s^{t^{\prime}}$ is a successor of $s^{t}$ (or $s^{t}$ itself), I write $s^{t^{\prime}} \succeq s^{t}$. The number of elements in $\mathcal{S}$ is $S$. The $S \times S$ transition matrix is denoted by $\pi$. With a slight abuse of notation, for $\sigma^{\prime} \succeq \sigma$, I write $\pi\left(\sigma^{\prime} \mid \sigma\right)$ to denote the conditional probability of $\sigma^{\prime}$ given $\sigma$.

I consider dynamic economic models where an equilibrium can be characterized by a system of semi-algebraic equalities and weak inequalities relating current-period exogenous and endogenous variables to endogenous and exogenous variables one period ahead. Examples of such conditions are individuals' Euler equations, firms' first-order conditions, and market-clearing equations for goods or financial assets. 
A subset $A \subset \mathbb{R}^{n}$ is a semi-algebraic subset of $\mathbb{R}^{n}$ if it can be written as the finite union and intersection of sets of the form $\left\{x \in \mathbb{R}^{n}: g(x)>0\right\}$ or $\left\{x \in \mathbb{R}^{n}: f(x)=0\right\}$ where $f$ and $g$ are polynomials in $x$ with coefficients in $\mathbb{R}$, that is, $f, g \in \mathbb{R}[x]$. Let $A \subset \mathbb{R}^{n}$ be a semi-algebraic set. A function $\theta: A \rightarrow \mathbb{R}^{m}$ is semi-algebraic if its graph $\left\{(x, y) \in A \times \mathbb{R}^{m}\right.$ : $y=\theta(x)\}$ is a semi-algebraic subset of $\mathbb{R}^{n+m}$. Blume and Zame (1993) and Kubler and Schmedders (2010a) discuss in detail the assumption of semi-algebraic fundamentals in finite exchange economies.

Current period endogenous variables are denoted by $z \in \mathbb{R}^{M}$. I assume that the system of inequalities characterizing equilibrium can be written as follows.

$$
h\left(\bar{s}, \bar{z}, z_{1}, \ldots, z_{S}\right)=0, \quad g(\bar{s}, \bar{z}) \geq 0
$$

where for each fixed $s \in \mathcal{S}, h$ and $g$ are continuous semi-algebraic functions. The arguments $(\bar{s}, \bar{z})$ denote the exogenous state and endogenous variables for the current period. The vector $z_{s} \in \mathbb{R}^{M}$ denotes endogenous variables in the subsequent period in state $s$. This is identical to the characterization in the example in Section 2, except that now one also has to consider $S$ possible exogenous shocks in the subsequent period.

A competitive equilibrium is then a process $\left(z\left(s^{t}\right)\right)$ such that for each $s^{t}$

$$
h\left(s_{t}, z\left(s^{t}\right), z\left(s^{t}, 1\right), \ldots, z\left(s^{t}, S\right)\right)=0, \quad g\left(s_{t}, z\left(s^{t}\right)\right) \geq 0
$$

It is useful to describe a competitive equilibrium not by infinite sequences but by a set that consists of at least all elements of the sequence but might also contain several equilibria at the same time.

Definition 1 An equilibrium set is a set $\mathcal{Z}=\mathcal{Z}_{1} \times \ldots \times \mathcal{Z}_{S} \subset \mathbb{R}^{M S}$, such that for all $\bar{s} \in \mathcal{S}$ and all $\bar{z} \in \mathcal{Z}_{\bar{s}}, g(\bar{s}, \bar{z}) \geq 0$, and there exist $\left(z_{1}, \ldots, z_{S}\right) \in \mathcal{Z}$ such that

$$
h\left(\bar{s}, \bar{z}, z_{1}, \ldots, z_{S}\right)=0 .
$$

Given the equilibrium equations (2), I define a backward operator to map variables in the next period into variables in the current period that are consistent with the equilibrium conditions. That is, given sets $K_{0}, K_{1}, \ldots, K_{S} \subset \mathbb{R}^{M}$, I define for each $\bar{s}$,

$$
\begin{array}{r}
\mathbf{B}_{\bar{s}}\left(K_{0},\left(K_{1}, \ldots, K_{S}\right)\right)=\left\{\bar{z} \in K_{0}: \exists z_{s} \in K_{s}, s=1, \ldots, S \text { such that } h\left(\bar{s}, \bar{z}, z_{1}, \ldots, z_{S}\right)=0\right. \\
g(\bar{s}, \bar{z}) \geq 0\} .
\end{array}
$$

Very roughly speaking, in the subsequent analysis this operator will play a role similar to the role of the Bellman operator in dynamic programming. The main difference is that it is defined on sets and not on functions and that in general one cannot prove any contraction or monotonicity properties for this operator. 


\subsection{Robust $\epsilon$-equilibria}

Given any $\epsilon \geq 0$, define an $\epsilon$-equilibrium set to be a set $\mathcal{Z}^{\epsilon}=\mathcal{Z}_{1}^{\epsilon} \times \ldots \times \mathcal{Z}_{S}^{\epsilon} \subset \mathbb{R}^{M S}$, such that for all $\bar{s} \in \mathcal{S}$ and all $\bar{z} \in \mathcal{Z}_{\bar{s}}^{\epsilon}, g(\bar{s}, \bar{z}) \geq 0$, and there exist $\left(z_{1}, \ldots, z_{S}\right) \in \mathcal{Z}^{\epsilon}$ such that

$$
\left\|h\left(\bar{s}, \bar{z}, z_{1}, \ldots, z_{S}\right)\right\| \leq \epsilon .
$$

The following is an abstract definition of a robust $\epsilon$-equilibrium set.

Definition 2 An $\epsilon$-equilibrium set $\mathcal{Z}^{\epsilon} \subset \mathbb{R}^{M S}$ is robust if it is closed and bounded and if for all $\bar{s} \in \mathcal{S}$,

$$
\mathbf{B}_{\bar{s}}\left(\mathbb{R}^{M},\left(\mathcal{Z}_{1}^{\epsilon}, \ldots, \mathcal{Z}_{S}^{\epsilon}\right)\right) \subset \mathcal{Z}_{\bar{s}}^{\epsilon} .
$$

The definition requires that for all endogenous variables in the $\epsilon$-equilibrium set which could realize in the subsequent period, all variables in the current period that are consistent with equilibrium must also lie in the set. I present a more intuitive economic interpretation of the concept below after characterizing robust $\epsilon$-equilibrium in terms of exact equilibrium. The following lemma provides the theoretical foundation for this.

Lemma 1 Suppose that there are (non-empty) closed and bounded sets $\left(K_{1}^{0}, \ldots, K_{S}^{0}\right)$ such that if one defines recursively, for each $\bar{s}$,

$$
K_{\bar{s}}^{i}=\mathbf{B}_{\bar{s}}\left(K_{\bar{s}}^{0},\left(K_{1}^{i-1}, \ldots, K_{S}^{i-1}\right)\right)
$$

each $K_{s}^{i}$ is non-empty and closed. Then there exists an equilibrium set with $\mathcal{Z}_{s} \subset K_{s}^{0}$ for all $s=1, \ldots, S$

Although the lemma follows directly from Duffie et al. (1994) I provide the proof for completeness. The proof also helps with understanding the subsequent analysis.

Proof of the Lemma. The main step of the proof consists in showing, by induction, that $K_{s}^{i} \subset K_{s}^{i-1}$ for all $i$ and for all $s$. By definition $K_{s}^{1} \subset K_{s}^{0}$ for all $s$. To show that if $K_{s}^{i} \subset K_{s}^{i-1}$, it must also be the case that $K_{s}^{i+1} \subset K_{s}^{i}$, observe that if for a given $\bar{s}, \bar{z}$, there exist $z_{s} \in K_{s}^{i}, s=1, \ldots, S$ such that $h\left(\bar{s}, \bar{z}, z_{1}, \ldots, z_{S}\right)=0, g(\bar{s}, \bar{z}) \geq 0$ then, since $K_{s}^{i} \subset K_{s}^{i-1}$, there must also exist $z_{s} \in K_{s}^{i-1}, s=1, \ldots, S$ satisfying this property, and hence $\bar{z}$ must lie in $K_{\bar{s}}^{i}$. Since the intersection of nested closed non-empty sets is non-empty, one can now define for each $s \in \mathcal{S}, \mathcal{Z}_{s}=\cap_{i=0}^{\infty} K_{s}^{i}$. Clearly the collection of sets $\left(\mathcal{Z}_{1}, \ldots, \mathcal{Z}_{S}\right)$ satisfy the conditions of an equilibrium set.

The lemma states that if one has candidate equilibrium sets $K^{0}$ and one can somehow prove that the recursively defined $K^{i}$ are non-empty for all $i$, then one can infer that each $K_{s}^{0}$ in fact contains an equilibrium set $\mathcal{Z}_{s}$. If one takes $K^{0}$ to be a robust $\epsilon$ equilibrium set, in order to apply the lemma one still somehow needs to verify that each $K^{i}$ is non-empty.

It is useful to do this by showing the existence of truncated equilibria. 
Definition 3 Given arbitrary sets $\left(Z_{1}, \ldots, Z_{S}\right), Z_{s} \subset \mathbb{R}^{M}$, define a $T$-truncated equilibrium with terminal condition $\left(Z_{1}, \ldots, Z_{S}\right)$ as a finite horizon process $\left(z\left(s^{t}\right)\right)_{t \leq T}$ such that for each $s^{t}$, $t \leq T-1$, the equilibrium conditions (3) hold and such that $z\left(s^{T}\right) \in Z_{s_{T}}$ for all terminal $s^{T}$.

The concept is closely related to the standard definition of equilibrium in truncated economies. The only difference is that in the final period, $T$, agents face prices, consumptions and investments prescribed by $Z$ and not, as in the standard concept, zero asset prices and no new trade. Showing existence of a T-truncated equilibrium with terminal condition turns out to be not much harder than showing existence of equilibria for truncated economies (which is part of standard existence proofs in these models). A sufficient condition for existence is typically that each $Z_{s}$ contains a continuous function, that there are constraints on trades which guarantee that it is never feasible to leave the specified state space and that budget sets are non-empty for all choices and prices. I illustrate this with an example in Section 5.1 below.

If for an arbitrary $\epsilon$-equilibrium set $\mathcal{Z}^{\epsilon}$, there exists a truncated equilibrium with terminal condition $\mathcal{Z}^{\epsilon}$ for all $T$, it is not guaranteed that the set contains an exact equilibrium since it is not guaranteed that the truncated equilibria take values in $\mathcal{Z}^{\epsilon}$. However, if the $\epsilon$-equilibrium set is robust, it is clear from the definition that the truncated equilibria (if they exist) must take values in the set. One can apply Lemma 1 and ensure that an exact equilibrium set must be contained in the $\epsilon$-equilibrium set. The following theorem states this formally.

Theorem 1 Suppose $\mathcal{Z}^{\epsilon}$ constitutes a robust $\epsilon$-equilibrium and that for each $T$ there exists a $T$-truncated equilibrium with terminal condition $\mathcal{Z}^{\epsilon}$. Then there exists an exact equilibrium set $\mathcal{Z}$ with $\mathcal{Z}_{s} \subset \mathcal{Z}_{s}^{\epsilon}$ for each $s \in \mathcal{S}$.

While the definition of robust $\epsilon$-equilibrium makes no mention of an exact equilibrium, this theorem allows for the following interpretation of robust $\epsilon$-equilibrium in terms of exact equilibrium.

One typically hopes that competitive equilibria in infinite horizon models are good approximations to equilibria in models with large finite horizons, and that these equilibria converge to the infinite equilibrium. In fact, one hopes that changes in exogenous variables in the far future have negligible effect on endogenous variables today. As Kubler and Schmedders (2005) show, $\epsilon$-equilibria can be interpreted as equilibria of a perturbed economy, i.e. equilibria of an economy with slightly different endowments or preferences. Robustness of an $\epsilon$-equilibrium set requires that no matter how exogenous variables in the future are (locally) perturbed, as long as the new equilibrium realizes in the $\epsilon$-equilibrium set, the effect on endogenous variables today must be no larger than the effect on endogenous variables at the date of the perturbation.

Note that a reverse interpretation is not possible. If up to some $T$, all endogenous variables realize in any $\epsilon$-equilibrium set, the only way that all equilibrium conditions from 
$T$ onwards hold exactly is that at the value of all endogenous variables at $T, z\left(s^{T}\right)$ already lies in an exact equilibrium set.

\subsection{Verification and existence of robust $\epsilon$-equilibria}

The main advantage of the concept is that (at least in principle) one can always check numerically whether a given semi-algebraic set constitutes a robust $\epsilon$-equilibrium. This follows directly from the so-called Tarski-Seidenberg principle and the quantifier elimination algorithm (see e.g. Bochnak et al. (1998, Chapter 5)). More precisely, there is an algorithm that decides for a given semi-algebraic set of $\epsilon$-equilibria if it is a robust $\epsilon$-equilibrium set ${ }^{2}$. If it is, it must contain an exact equilibrium. This raises the question of whether one should expect robust $\epsilon$-equilibrium sets to exist and to be semi-algebraic.

\subsubsection{Existence of robust $\epsilon$-equilibrium}

Suppose from the economic model one can find a priori bounds on all endogenous equilibrium variables (the example below shows this is usually not very difficult, these bounds typically arise from non-negativity constraints in consumption, market-clearing etc.) Let $K^{0}$ satisfy these bounds and, without loss of generality, impose the bounds in the equilibrium inequality $g() \geq$.0 .

It is clear that, if $K^{0}$ is semi-algebraic, the constructed $K_{s}^{i}$ are semi-algebraic for all $i=1, \ldots$ and each $s$. Fixing an $\epsilon>0$, there must now exist a sufficiently large $i$ such that $K^{i}$ actually constitutes an $\epsilon$-equilibrium set. By boundedness of $K^{0}$, for each $\delta>0$ there must be an $i$ such that for all $s$, if $z \in K_{s}^{i}$, there must be a $\tilde{z} \in K_{s}^{i+1}$ with $\|z-\tilde{z}\|<\delta$. For each $\epsilon$, there must be a $\delta$, so that if $h\left(\bar{s}, \tilde{z}, z_{1}, \ldots, z_{S}\right)=0$ then $\left\|h\left(\bar{s}, z, z_{1}, \ldots, z_{S}\right)\right\| \leq \epsilon$ whenever $\|z-\tilde{z}\|<\delta$.

The construction also implies that $K^{i}$ is robust: If there existed some $\bar{s}, \bar{z}$ with $\bar{z} \in$ $\mathbf{B}_{\bar{s}}\left(\mathbb{R}^{M},\left(K_{1}^{i}, \ldots, K_{S}^{i}\right)\right)$ but $\bar{z} \notin K_{\bar{s}}^{i}$, clearly by Lemma 1 , we must have $\bar{z} \notin K_{\bar{s}}^{0}$. But this is impossible because the inequality $g() \geq$.0 imposes $\bar{z} \in K_{\bar{s}}^{0}$ by constructions.

\section{A recursive formulation}

So far, the analysis has been conducted for abstract $(\epsilon)$ equilibrium sets. However, while it is easy to compute one 'recursive' approximate equilibrium, researchers typically do not explicitly compute entire sets of equilibria. In this Section, I use the theoretical results from above to show that it is possible to verify that a recursive $\epsilon$-equilibrium is close to an exact (not necessarily recursive) competitive equilibrium.

For this, I need to impose a bit more structure on the abstract economy and define a recursive $\epsilon$-equilibrium. As in Section 2, I write the vector of endogenous variables as $z=$

\footnotetext{
${ }^{2}$ It is well known that quantifier elimination is hopelessly inefficient. I introduce more tractable methods for this below.
} 
$\left(\theta_{-}, \eta\right)$, with $\theta_{-}$being the 'endogenous state'. The relevant endogenous state space is $\Theta=$ $\left(\Theta_{1}, \ldots, \Theta_{S}\right)$ where each $\Theta_{s} \subset \mathbb{R}^{D}$ depends on the underlying model and is determined by the payoff-relevant pre-determined endogenous variables; that is, by variables sufficient for the optimization of individuals at every date-event, given the prices. If $\Theta$ is the 'endogenous state space' there must exist set-valued functions $\rho_{s}: \Theta_{s} \rightrightarrows \mathbb{R}^{M-D}$ such that each $\mathcal{Z}_{s}=$ $\operatorname{graph}\left(\rho_{s}\right)$ for all $s \in \mathcal{S}$.

The function $h($.$) typically uniquely determines \theta_{-s}$ for each shock $s$, as a function of $\bar{z}$. In the simplest example, the beginning-of-period portfolio holding is the endogenous state and this is equal to the last period's choices across agents. I illustrate this point in the next section.

The value of the state variables $s_{0} \in \mathcal{S}, \theta_{-}(0) \in \Theta_{s_{0}}$ in period 0 is called 'initial condition' and is part of the description of the economy. It will often be useful to make this explicit. In particular, I often want to require that an equilibrium set describes a family of equilibria arising from different initial conditions in a set of $\theta_{-}(0)$ that contains an open set. Through this requirement, the state-space $\Theta$ is partly specified exogenously, but it is of course endogenous in the sense it must contain all realizations of $\theta_{-}$that occur in equilibrium. In some models with exogenous constraints on trades, $\Theta$ can be taken as exogenous since the realizations of $\theta_{-}$are predetermined through these restrictions. This applies for example in models with asset markets and short-sale constraints on these assets. For the purpose of this section, it will be useful to assume that there are sufficient constraints on trades that ensure that in fact $\Theta$ is specified exogenously. I will give an example below where this is not the case and show that it is without loss of generality to assume that agents face trading constraints that are never binding in equilibrium.

A recursive $\epsilon$-equilibrium consists of sets $\hat{\Theta}_{s}$, and functions $\hat{\rho}_{s}: \hat{\Theta}_{s} \rightarrow \mathbb{R}^{M-D}, s \in \mathcal{S}$ such that if $\mathcal{Z}_{s}^{\epsilon}=\operatorname{graph}\left(\hat{\rho}_{s}\right)$ for all $s \in \mathcal{S}$, then $\mathcal{Z}^{\epsilon}$ constitutes an $\epsilon$-equilibrium set. Note that the comment made about the exogeneity of $\Theta$ also applies to $\hat{\Theta}$. Again I will assume that $\hat{\Theta}$ is given through the description of the economy and that therefore $\Theta_{s}=\hat{\Theta}_{s}$ for all $s \in \mathcal{S}$. From now on, I will also assume that $\hat{\rho}_{s}$ is a continuous semi-algebraic function (which implies that $\hat{\Theta}_{s}$ is a semi-algebraic set) for all $s \in \mathcal{S}$.

It is easy to see that $\hat{\rho}_{s}$ itself, since it is a function, will never describe a robust $\epsilon$ equilibrium set (unless it is an exact equilibrium). The first step is therefore to create a function strip around $\hat{\rho}$ that describes an entire set of $\epsilon$-equilibria.

\subsection{Constructing robust $\epsilon$-equilibrium sets}

For a given approximate recursive equilibrium $\left(\hat{\Theta}_{s}, \hat{\rho}_{s}\right)_{s \in \mathcal{S}}$ and fixed $\delta>0$, I take as a candidate $\epsilon$-equilibrium set

$$
\mathcal{Z}_{s}^{\epsilon}=\left\{\left(\theta_{-}, \eta\right): \theta_{-} \in \hat{\Theta}_{s}, \quad\left\|\eta-\hat{\rho}_{s}\left(\theta_{-}\right)\right\| \leq \delta\right\}, \quad s \in \mathcal{S} .
$$

Note that the exact relation between $\delta$ and $\epsilon$ in this definition is not important for what follows. What is important is that if, for sufficiently small $\delta, \mathcal{Z}^{\epsilon}$ contains an exact compet- 
itive equilibrium then $\mathcal{Z}^{\epsilon}$ should also be a robust $\epsilon$-equilibrium set. At this abstract level this is not entirely clear. This should certainly be trued if close to the exact equilibrium the backward operator is monotone but actually turns out to hold more generally. In Section 5, I illustrate this point in detail.

Given the above analysis, in order to verify robustness, one now needs to verify that for all $s \in \mathcal{S}$,

$$
\mathbf{B}_{s}\left(\mathbb{R}^{M},\left(\mathcal{Z}_{1}^{\epsilon}, \ldots, \mathcal{Z}_{S}^{\epsilon}\right)\right) \subset \mathcal{Z}_{s}^{\epsilon} .
$$

However, this neglects the fact that one would like the recursive $\epsilon$-equilibrium to be close to an exact equilibrium for all $\theta_{-} \in \hat{\Theta}_{s}, s \in \mathcal{S}$. But since I assumed that $\Theta$ is given exogenously through constraints on trades, it is without loss of generality to impose that the inequalities $h() \geq$.0 ensure that $\theta_{-s}$ always realize in $\Theta_{s}$. Therefore the definition of robust equilibrium is now equivalent to the following, perhaps more intuitive concept. Define for each $s \in \mathcal{S}$, $\mathcal{Y}_{s}=\left\{z=\left(\theta_{-}, \eta\right) \in \mathbb{R}^{M}: \theta_{-} \in \hat{\Theta}_{s}\right\}$ and require for $\mathcal{Z}^{\epsilon}$ that for each $s \in \mathcal{S}$,

$$
\mathbf{B}_{s}\left(\mathcal{Y}_{s},\left(\mathcal{Z}_{1}^{\epsilon}, \ldots, \mathcal{Z}_{S}^{\epsilon}\right)\right) \subset \mathcal{Z}_{s}^{\epsilon} .
$$

In other words, if endogenous variables in next period lie within some $\delta$ of $\hat{\rho}$ all endogenous variables in this period must also lie within $\delta$ of $\hat{\rho}$. Of course, Theorem 1 now needs to be slightly modified and one needs to verify that truncated equilibria exist for all initial conditions in $\hat{\Theta}$. The rest of the argument then remains the same.

If $\mathcal{Z}^{\epsilon}$ is robust, and the conditions of Theorem 1 are satisfied, it must contain an exact equilibrium, i.e. the computed approximation must be within $\delta$ of an exact equilibrium for all values of the state. Note that these are absolute errors. Alternatively, we could have defined

$$
\mathcal{Z}_{s}^{\epsilon}=\left\{\left(\theta_{-}, \eta\right): \theta_{-} \in \hat{\Theta}_{s}, \quad \max _{i}\left|\frac{\eta_{i}}{\hat{\rho}_{s i}\left(\theta_{-}\right)}-1\right| \leq \delta\right\}
$$

to obtain relative errors. The exposition in this section uses absolute errors, while I will use relative errors in some of the examples below.

The advantage of working in a recursive framework is that one can formulate Condition (5) as a constrained optimization problem. Given a fixed $\delta>0$, I consider the following constrained optimization problem for each $s \in \mathcal{S}$,

$$
\begin{aligned}
\max _{\theta_{-} \in \hat{\Theta}_{s}, \epsilon, \eta}\left\|\eta-\hat{\rho}\left(\theta_{-}\right)\right\| \text {s.t. } & \|\epsilon\| \leq \delta \\
& h\left(s, \theta_{-}, \eta,\left(\theta_{-1}, \hat{\rho}_{1}\left(\theta_{-1}\right)+\epsilon_{1}\right), \ldots,\left(\theta_{-S}, \hat{\rho}_{S}\left(\theta_{-S}\right)+\epsilon_{S}\right)\right)=0 \\
& g\left(s, \theta_{-}, \eta\right) \geq 0
\end{aligned}
$$

It is easy to see that, if the optimal value of this problem lies below $\delta$, the set $\mathcal{Z}^{\epsilon}$ as defined in (4) is a robust $\epsilon$-equilibrium set.

Since the optimization problem (6) is not a convex programming problem one can generally not find the global maximum. However, in the semi-algebraic case, it is a different matter. I assume that $h, g$ and $\hat{\rho}$ are continuous semi-algebraic functions and that $\hat{\Theta}_{s}$ are 
closed semi-algebraic sets. It follows from Proposition 2.1.8. of Bochnak et al. (1998) that one can solve the maximization problem (6) by solving a series of polynomial optimization problems and verifying additional polynomial inequalities. See also Kubler and Schmedders (2010a) for a detailed description of this point in finite economies. To simplify notation I will assume from now on that $h, g$ and $\hat{\rho}$ are already polynomial functions. In the applications below, the equilibrium conditions can be rewritten directly as polynomial functions and $\hat{\rho}$ is polynomial to start with.

\subsection{Solving the maximization problem (6)}

Under the assumption that the Kuhn-Tucker conditions are necessary and have finitely many isolated solutions, algorithms designed to find all solutions to polynomial equations (see Sturmfels (2002) for an overview) can be used to find all critical points and, by comparing them, one can find the globally optimal solution to system (6). However, this 'brute force' approach is extremely inefficient.

It turns out to be much more efficient to use semi-definite programming and so called sum-of-squares relaxation to solve the polynomial optimization problem. In the following, I briefly explain the basic idea of the method.

\subsubsection{Sum-of-squares relaxations}

In the last decade, big advances have been made in polynomial optimization - see Laurent (2009) for an overview. The basic idea, (which is nicely explained in detail in e.g. Parrilo (2003)), is as follows.

A polynomial $p \in \mathbb{R}[x]$ is said to be a sum of squares (of polynomials) if it can be written as

$$
p=\sum_{j=1}^{m} u_{j}^{2} \text { for some } u_{j} \in \mathbb{R}[x] .
$$

Clearly, if for a polynomial $p$, there exists a number $\gamma$, such that $p-\gamma$ is a sum of squares, then $\gamma$ is a lower bound for $p(x)$ for any $x$.

If the degree of $p$ is $d$, in order for it to be a sum of squares, there have to exist $u_{j}$ which are of degree $d / 2$. The main insight is now that one can use semi-definite programming to search over all polynomials of degree $d / 2$ to establish that $p$ is the sum of squares. The polynomial $p$ can be written as a quadratic form of all the monomials of degree less than or equal to $d$, i.e. let $z=\left[1, x_{1}, x_{2}, \ldots, x_{n}, x_{1}^{2}, x_{1} x_{2}, \ldots, x_{n}^{d}\right]$ be the vector of all such monomials. Then there must exist a positive-definite matrix $Q$ with

$$
p(x)=z^{T} Q z .
$$

This matrix can be found using semi-definite programming (see Parrilo (2003) for details).

Following the same idea, but slightly more complicated, now consider the constrained optimization problem

$$
\min f(x) \text { s.t }
$$




$$
g_{1}(x)=\ldots=g_{m}(x)=0, \quad h_{1}(x) \geq 0, \ldots, h_{l}(x) \geq 0 .
$$

If there exist a number $\gamma$, arbitrary polynomials $q_{1}, \ldots, q_{m} \in \mathbb{R}[x]$ and sum-of-squares polynomials $r_{1}, \ldots, r_{l} \in \mathbb{R}[x]$ such that

$$
p:=f-\gamma-\sum_{i=1}^{m} g_{i} q_{i}-\sum_{i=1}^{l} h_{i} r_{i}
$$

is a sum-of-squares, then clearly $f(x) \geq \gamma$ for all $x$ satisfying $g(x)=0, h(x) \geq 0$. So again, $\gamma$ is a lower bound for the minimization problem. As before, given a candidate $p$, semi-definite programming can be used to efficiently check if $p$ is a sum-of-squares.

It is quite complicated to derive conditions on $g$ and $h$ that ensure that the converse holds, i.e. if $\gamma$ solves the minimization problem, one can achieve the sum-of-squares representation. The Positivstellensätze by Schmüdgen and by Putinar (see Laurent (2009, Theorem 3.16)) provide an answer - it is beyond the scope of this paper to discuss this further.

More important for this paper is the fact that it is not possible to find good bounds on the degree of $q$ and $r$ and therefore on the degree of $p$ in equation (7). While one can still use semi-definite programming to determine for which $\gamma$ the term in the equation can be written as a sum of squares, one has no a priori-bound on the degree and therefore has to experiment with different values. Fixing the maximal degree of the polynomial $p$, semi-definite programming can be used to determine efficiently if polynomials $q$ and $r$ of appropriate degree exist. Waki et al. (2006) provide a way to exploit sparseness in the polynomial problem so that the method is applicable to interesting problems. They report solving (sparse) problems with several hundred variables.

In Section 5 below, I use a matlab implementation by the authors called SparsePOP, described in Waki et al. (2008) to solve the relatively small problems that arise from the applications. The package produces a lower bound for the problem, $\gamma$, from the solution of the semi-definite program, as well as an approximate solution (the minimizer) to the polynomial problem. If these values coincide, a true minimum has been found. If $\gamma$ is smaller than the value of $f$ at the approximate solution, $\gamma$ is still a lower bound for the problem but neither $\gamma$ nor the value of $f$ might be the true minimum.

It is important to understand that one cannot always guarantee that the algorithm finds the global minimum. There are essentially two reasons for this. First, it turns out that solving the resulting semi-definite program is a difficult numerical problem and the solver might fail to find a solution (see Waki et al. (2006) for a extensive discussion of this problem and some possible remedies). More importantly, to use SparsePOP one needs to specify the degree of relaxation (the parameter 'param.relaxOrder' ), which is a bound on $d / 2$ where $d$ is the degree of the polynomial $p$ in Equation (7). As explained above, one cannot say a priori how large this should be. In the examples, below I try the values 3, 4, 5 and 6 . Large values generally lead to severe numerical problems. Obviously, even if there exist $\gamma$ and 
polynomials $r$ and $u$ such that $p$ can be written as the sum of squares, it is not guaranteed that it will be of degree 8 or less.

However, the output of the algorithm is always a lower bound on the true value of the minimization problem. For my purposes, it is irrelevant what the true value of the problem is. In order to determine if the error set $\mathcal{E}$ is empty for a given $\delta$, one actually does not have to solve the maximization problem (6). It suffices that $\delta$ provides an upper bound for the problem. So if the software package finds a $\gamma<\delta$ for which the problem can be written as a sum-of-squares, existence of a robust equilibrium within $\delta$ of the candidate equilibrium is proven. If the software package does not find such a $\gamma$, nothing can be said about the robustness of the candidate solution.

Note that this is a numerical algorithm, i.e. an approximate numerical solution is computed using floating point arithmetic and rounding errors could potentially lead to problems. Peyrl and Parrilo (2008) develop an algorithm that computes an exact algebraic solution if coefficients are rational.

\subsubsection{Practical Considerations}

As I will explain in the next section, it is important to formulate the optimization problem so that the degree of the polynomials is relatively low and it is important to find good bounds on the variables. For many interesting economic applications, this might not always be possible and it might not be feasible to use existing implementations of these methods to solve the polynomial optimization problem. From a practical perspective, the insights from this section are useful nevertheless. There are various efficient methods to find local minima to the optimization problem (6) and, while this obvious cannot lead to a guarantee that the computed approximation is close to an exact equilibrium, it can be a useful necessary check. In particular, independently of the economy being semi-algebraic, the maximization problem can be viewed as a programming problem with equilibrium constraints. There is a large literature on these problems (see e.g. Luo et al. (1996)) and reliable software to solve large-scale problems (see e.g. Su and Judd (2008) for an application of these methods to economics).

\section{Example: Stochastic overlapping generations}

In this section, I illustrate the method using a simple stochastic olg economy. This is the natural extension of the model considered in Section 2 in an environment with uncertainty. Agents live for three periods, there is a single good, a single agent per generation and a Markov chain determines endowments over the life cycle. I assume that there is no production and that financial markets are incomplete ${ }^{3}$ and that there is a single bond

\footnotetext{
${ }^{3}$ In an earlier version of the paper, I considered the case of complete markets. This makes the analysis slightly easier. Results are similar and available upon request.
} 
available for trade. The main purpose of this section is to illustrate the theoretical results above. Clearly there is a trade-off between the model and notation being extremely simple and the model being interesting, i.e. somewhat realistic or similar to models used in other applications.

At each date-event a single individual commences his economic life; he lives for three periods. An individual is identified by the date event of his birth, $\sigma=\left(s^{t}\right)$. The age of an individual is $a=1,2,3$; he consumes and has endowments at all nodes $s^{t-1+a} \succeq s^{t}$, $a=1,2,3$. An agent's individual endowments are a function of the shock and his age alone, i.e. for all $a=1,2,3, e^{s^{t}}\left(s^{t-1+a}\right)=\mathbf{e}_{a}\left(s_{t-1+a}\right)$ for some function $\mathbf{e}_{a}: \mathcal{S} \rightarrow \mathbb{R}_{+}$.

The agent has an intertemporal time-separable expected utility function.

$$
U^{\sigma}(c)=\sum_{a=1}^{3} \sum_{s^{t-1+a} \succeq \sigma} \pi\left(s^{t-1+a} \mid \sigma\right) u_{a}\left(c\left(s^{t-1+a}\right), s_{t-1+a}\right)
$$

The Bernoulli utility $u$ depends on the age and the current shock alone.

At each $s^{t}$, there is a single risk-free bond in zero net supply available for trade. Its price is denoted by $q\left(s^{t}\right) \in \mathbb{R}_{+}$and agent $\sigma^{\prime}$ 's bond-holding is $\theta^{\sigma}\left(s^{t}\right) \in \mathbb{R}$. Agents might face a borrowing constraint of the form $\theta^{\sigma}\left(s^{t}\right) \geq b$, for some $b<0$.

At the root node, $s_{0}$, there are individuals of all ages $s^{-1}, s^{-2}$ with initial wealth $\theta^{s^{-a}}\left(s^{-1}\right)$. These determine the 'initial condition' of the economy.

It will turn out to be useful to write $c_{a}\left(s^{t}\right)$ and $\theta_{a}\left(s^{t}\right)$ to denote consumption and portfolios of the agent born at $s^{t-1+a}$.

A competitive equilibrium is a collection of prices and choices of individuals such that markets clear and agents optimize, i.e. a sequence $\left(q\left(s^{t}\right),\left(\theta_{a}\left(s^{t}\right), c_{a}\left(s^{t}\right)\right)_{a=1,2,3}\right)_{s^{t} \in \Sigma}$ such that for all nodes $s^{t} \in \Sigma$ the following holds:

- Market clearing:

$$
\sum_{a=1}^{3} \theta_{a}\left(s^{t}\right)=0
$$

- At each $s^{t}$, individual $\sigma=s^{t}$ maximizes utility:

$$
\begin{gathered}
\left(c^{\sigma}, \theta^{\sigma}\right) \in \underset{c \geq 0, \theta}{\arg \max } U^{\sigma}(c) \text { s.t. } \\
c\left(s^{t}\right)-\mathbf{e}_{1}\left(s_{t}\right)+q\left(s^{t}\right) \theta\left(s^{t}\right) \leq 0, \quad \theta\left(s^{t}\right) \geq b \\
c\left(s^{t+1}\right)-\mathbf{e}_{2}\left(s_{t+1}\right)+q\left(s^{t+1}\right) \theta\left(s^{t+1}\right)-\theta\left(s^{t}\right) \leq 0, \quad \theta\left(s^{t+1}\right) \geq b \\
c\left(s^{t+2}\right)-\mathbf{e}_{3}\left(s_{t+2}\right)-\theta\left(s^{t+1}\right) \leq 0 \\
\text { for all } s^{t+1} \succeq \sigma \text { and all } s^{t+2} \succeq s^{t+1}
\end{gathered}
$$

Optimality conditions for initially alive agents, $s^{-1}$ and $s^{-2}$ are analogous. 
As in the deterministic example in Section 2, the natural endogenous state space of this economy consists of beginning of period bond-holdings of the middle aged. Define $c=\left(c_{1}, c_{2}, c_{3}\right)$ to be consumption across agents alive in the current period, $\theta=\left(\theta_{1}, \theta_{2}\right)$ to be new portfolio choices and $\kappa=\left(\kappa_{1}, \kappa_{2}\right) \in \mathbb{R}_{+}^{2}$ to be the multipliers associated with the borrowing constraint, as well as $\theta_{-}$to be the beginning of period wealth of the middle aged and the old. Let $z=\left(\theta_{-}, q, c, \theta, \kappa\right)$ denote the vector of endogenous variables in a given period.

It is well known that under the assumption that $U^{h}$ is differentiable, strictly increasing, strictly quasi-concave and satisfies an Inada-condition, the first order conditions are necessary and sufficient for agent optimality. As in Section 2, the equilibrium equations consist of the first order conditions, budget equations, market-clearing and the equations that determine cash-at-hand in the next period, given choices today.

In this example, the state space does not depend on the shock. Note that if $b=-\infty$ and there are no constraints on trades, the set $\Theta$ is completely endogenous. Therefore, the previous analysis has to be slightly modified. One can view a recursive $\epsilon$-equilibrium of an economy without constraints that is given by $\hat{\rho}_{s}: \hat{\Theta} \rightarrow \mathbb{R}^{M-D}$ as an $\epsilon$-equilibrium of an economy where the young agent faces the constraint $\theta_{s} \in \hat{\Theta}$, but the constraint is simply never binding. Through the error set, one then needs to verify that this $\epsilon$-equilibrium is close to an exact equilibrium with the same constraints in which these constraints are also never binding. In practice, I therefore check if a $\delta$-strip around the computed $\epsilon$-equilibrium is a robust $\epsilon$-equilibrium set for the economy with constraints and then show that in the exact equilibrium these constraints are never binding.

In order to apply the main result of the paper, Theorem 1, one first needs to establish existence of truncated equilibria with terminal conditions. To prove existence of competitive equilibria in these models, one typically first proves that an equilibrium exists for all finitely truncated economies and then takes the limit. The result needed here is very similar.

\subsection{Existence of truncated equilibrium}

In this section, I prove that in the olg model truncated equilibria with initial conditions $Z$ always exist if the set $Z$ contains the graph of a continuous approximate policy function. Let $\hat{\rho}_{q}$ denote the approximate pricing function and $\hat{\rho}_{\theta}$ the approximate policy function of the middle aged. Let $\underline{c}>0$ denote a lower bound of an agents' consumption in any equilibrium. This is some positive number determined by the fact that endowments are strictly positive and that agents are finitely lived and their utility satisfies an Inada condition.

As mentioned above, the existence of a truncated equilibrium is shown for a slightly modified economy where all agents face additional trading constraints of the form $\theta \geq b$. By market clearing, this implies $b \leq \theta_{1} \leq-b$ and one obtains a compact state space $\hat{\Theta}=[b,-b]$.

In this setup, one obtains the following lemma. 
Lemma 2 Suppose $\hat{\rho}$ is continuous and that for any $s \in \mathcal{S}$ and any $\theta_{-} \in \hat{\Theta}, \mathbf{e}_{2}(s)+\theta_{-}-$ $\hat{\rho}_{q}\left(\theta_{-}, s\right) \cdot \hat{\rho}_{\theta}\left(\theta_{-}, s\right)>\underline{c}$. Then for all initial conditions $s_{0}, \theta_{-}\left(s_{0}\right) \in \hat{\Theta}$ and for any $T$, there exists a $T$-truncated competitive equilibrium with terminal condition $\hat{\rho}$.

Although the proof of the lemma is a standard application of Kakutani's theorem (see e.g. Kubler and Polemarchakis (2004)), I present a detailed outline. The only difficulties lie in making assumptions that ensure that agents' budget sets are non-empty and in modifying the problems of agents alive at $T-1$ and $T$; I discuss these in detail.

Fix initial conditions $s_{0}, \theta_{-}\left(s_{0}\right) \in \hat{\Theta}$. Each agent in the $T$ horizon economy who is not active at $T$ takes prices as given and a standard argument shows that his best response is continuous for strictly positive prices. At each node $s^{t}, t<T$, there is a price player that takes choices at the node as given and solves

$$
\max _{(p, q) \in \Delta_{\eta}^{2}} p\left(c_{1}\left(s^{t}\right)+c_{2}\left(s^{t}\right)+c_{3}\left(s^{t}\right)-e_{1}\left(s_{t}\right)-e_{2}\left(s_{t}\right)-e_{3}\left(s_{t}\right)\right)+q\left(\theta_{1}\left(s^{t}\right)+\theta_{2}\left(s^{t}\right)\right),
$$

where $\Delta_{\eta}^{2}=\{(p, q): p+q=1, p \geq \eta, q \geq \eta\}$ denotes the two dimensional truncated simplex. His choices are upper-hemi continuous and convex valued in choices of all agents.

Given choices of all agents, let $\bar{q}\left(s^{T}\right)=\hat{\rho}_{q}\left(\theta^{1}\left(s^{T-1}\right)\right)$ and let $\bar{\theta}\left(s^{T}\right)=\hat{\rho}_{\theta}\left(\theta^{1}\left(s^{T-1}\right)\right)$. Clearly this is continuous and non-empty for all admissible choices of agents.

Finally, all agents born at period $T-1$ at node $s^{T-1}$, take as given $\bar{q}\left(s^{T}\right)$ and $\bar{\theta}\left(s^{T}\right)$, as well as prices at $s^{T-1}$. Define their consumption at $T$ as a function of savings at $T-1$ as

$$
c(\theta)=\max \left(\left(1+\frac{\theta}{2 b}\right) \frac{c}{4}, e_{2}\left(s_{T}\right)+\theta-\bar{q}\left(s^{T}\right) \cdot \bar{\theta}\left(s^{T}\right)\right) .
$$

This definition ensures that, independently of $\left(\bar{q}\left(s^{T}\right), \bar{\theta}\left(s^{T}\right)\right)$, positive consumption is always feasible. It is then standard to show that best responses are continuous and non-empty since the budget sets are continuous and non-empty in prices and in $\bar{q}, \bar{\theta}$. Kakutani's theorem ensures the existence of a fixed point of the Cartesian product of the best-responses - it is standard to show that for sufficiently small $\eta>0$, this is a T-truncated equilibrium according to the above definition. In particular, at the fixed point, it is guaranteed that $c^{s^{T-1}}\left(s^{T}\right)=e_{2}\left(s_{T}\right)+\theta\left(s^{T-1}\right)-\bar{q}\left(s^{T}\right) \cdot \bar{\theta}\left(s^{T}\right)$ by the definition of $\underline{c}$ and the assumptions on $\hat{\rho}$.

The main insight of the proof is that, if for a given $T$-horizon economy one can show that agents' choices are continuous in prices, if one imposes a continuous policy function at $T$ which allows for positive consumption at some values of the specified state space, and if one imposes constraints on trades that ensure that agents' actions will never result in a state outside of the specified space $\hat{\Theta}$, then the only modification of the standard existence proof consists of the assumption that agents take choices at $T$ as given. The method of proof can be applied to a large variety of models where competitive equilibrium exists. 


\subsection{Examples}

As explained in the introduction, this paper is not about how to compute approximate equilibrium. For the simple model in this section, there are several reliable methods to do so. I use the time-iteration algorithm which is explained in detail in Kubler and Krueger (2004) for a model with overlapping generations and production. I first revisit the deterministic example from Section 2 and then consider an example with uncertainty. Throughout, I assume that for each $a$ and $s, u_{a}(c, s)=-\beta^{a} c^{1-\sigma}, \beta>0$, i.e. preferences exhibit constant relative risk aversion with a coefficient of relative risk aversion of $\sigma>1$.

\subsubsection{Example 1: A deterministic economy}

To fix ideas, I first reconsider the example from Section 2. Inspecting the equilibrium equations (1), one notices that, in order to solve the constrained maximization problem (6), one does not need to know the entire policy function but in fact only the consumption policies. It is therefore useful to approximate these separately, i.e. instead of plugging the portfolioand pricing functions into the budget constraints, and so obtaining an approximating polynomial for consumption, it makes more sense to approximate consumption directly, solve the constrained maximization problem using only this function and then ask what it implies for portfolios and prices. Concretely, for the two specifications it turns out that consumption policies are actually better behaved then portfolios and can be well approximated by polynomials of degree 3 .

In order to verify that a candidate solution is close to an exact equilibrium one now has to go through several steps: (1) Given a policy function of the middle aged, $\hat{\rho}_{c}($.$) , the$ constrained maximization problem to determine if this can be part of a robust $\epsilon$-equilibrium is as follows.

$$
\begin{aligned}
& \max _{\epsilon, \theta_{-} \in \hat{\Theta}, q, \theta} \pm\left(e_{2}+\theta_{-}+q \cdot \theta-\hat{\rho}_{c}\left(\theta_{-}\right)\right) \text {s.t. } \\
& -\tilde{q}^{1 / 3}\left(\epsilon+\hat{\rho}_{c}(\theta)\right)+\beta^{1 / 3}\left(e_{1}-\tilde{q}^{3} \theta\right)=0, \\
& -\tilde{q}^{1 / 3}\left(e_{3}-\theta\right)+\beta^{1 / 3}\left(e_{2}+\tilde{q}^{3} \theta+\theta_{-}\right)=0, \\
& \theta \in \hat{\Theta}, \quad q \geq 0, \quad-\delta \leq \epsilon \leq \delta
\end{aligned}
$$

where \pm indicates that, firstly, the positive objective function is maximized and, secondly, the negative objective function is maximized.

Note that it is useful to substitute in budget constraints and to write the system using $\tilde{q}=q^{1 / \sigma}$. In its original formulation, the system of constraints would contain the term $q\left(\epsilon+\hat{\rho}_{c}(\theta)\right)^{3}$ - with $\hat{\rho}_{c}$ being a polynomial of degree 3 that would result in a polynomial of degree 7 and potentially cause numerical problems. Note also that, although in its original formulation, the example did not include constraints on trades, I add the constraint $\theta \in \hat{\Theta}$. Since the polynomial function $\hat{\rho}_{c}$ is defined on all of $\mathbb{R}$, but only makes sense on $\hat{\Theta}$, this cannot be avoided. For candidate solution (1) and $\delta=10^{-4}$, SparsePOP returns an upper bound of $4.3 \times 10^{-5}$ for the problem. As a next step, (2), one has to check what a deviation 
of $10^{-4}$ in the consumption policy implies for prices and portfolios. For this, one can use SparsePOP to solve $\max _{\epsilon, \theta_{-} \in \hat{\Theta}, q, \theta} \pm\left(\theta-\hat{\rho}_{\theta}\left(\theta_{-}\right)\right)$and $\max _{\epsilon, \theta_{-} \in \hat{\Theta}, q, \theta} \pm\left(q-\hat{\rho}_{q}\left(\theta_{-}\right)\right)$subject to the same constraints as above. For Solution (1), SparsePOP returns an upper bound of $3.2 \times 10^{-4}$. Finally, in step (3), one now has to verify that, if portfolios stay in this region, the additional superficial constraint $\theta \in \hat{\Theta}$ never binds, i.e. in this case, for Solution (1), one needs to check that for all $\theta_{-} \in[-6,0], \hat{\rho}\left(\theta_{-}\right)-3.2 \times 10^{-4}>-6$ and $\hat{\rho}\left(\theta_{-}\right)+3.2 \times 10^{-4}<0$ - which is the case.

In comparison, for Solution (2), Steps (1) and (2) above also yield good results. In this case, one obtains that the portfolio policy is within $10^{-2}$ of $\hat{\rho}\left(\theta_{-}\right)$. However, with this, Step (3) fails since $\hat{\rho}\left(\theta_{-}\right)+10^{-2}$ lies outside of $\hat{\Theta}$ at $\theta_{-}=-5$.4. The method fails to verify that this approximate solution is close to an exact equilibrium - as I show in Appendix A it is not.

\subsubsection{Example 2: Endowment uncertainty}

The introduction of uncertainty potentially causes numerical problems. If one continues to use the consumption policy-function for CRRA utility and an economy with $S$ exogenous shocks, one has to solve $s=1, \ldots, S$ problems of the form

$$
\begin{aligned}
& \max _{\left(\epsilon_{1}, \ldots, \epsilon_{S}\right), \theta-\in \Theta, q, \theta} \pm\left(e_{2}(s)+\theta+q \theta-\hat{\rho}_{c}\left(\theta_{-}\right)\right) \quad \text { s.t. } \\
& -q \frac{1}{\left(e_{1}(s)-q \theta\right)^{\sigma}}+\sum_{s^{\prime}=1}^{S} \pi\left(s^{\prime} \mid s\right) \beta \frac{1}{\left(\epsilon_{s^{\prime}}+\hat{\rho}_{c^{1}}\left(\theta, s^{\prime}\right)\right)^{\sigma}}=0 \\
& -q \frac{1}{\left(e_{2}(s)+q \theta+\theta-\right)^{\sigma}}+\sum_{s^{\prime}=1}^{S} \pi\left(s^{\prime} \mid s\right) \beta \frac{1}{\left(e_{3}(s)-\theta\right)^{\sigma}}=0 \\
& q \geq 0, \theta \in \hat{\Theta} \text { and }-\delta \leq \epsilon_{s^{\prime}} \leq \delta, \quad \text { for all } s^{\prime} \in \mathcal{S} .
\end{aligned}
$$

In this formulation, the equilibrium constraints are not polynomial but, of course, if $\sigma$ is rational, one can obtain polynomial expressions by multiplying out. Even for very simple examples with $\sigma=3$ and two shocks, if the approximate consumption function is cubic this leads to polynomials of degree 13. This is not feasible for SparsePOP. Instead, for a given current shock $s$, it is useful to consider the functions

$$
\begin{gathered}
\hat{\rho}_{m^{1}}\left(\theta_{-}\right)=\left(\sum_{s^{\prime}=1}^{S} \pi\left(s^{\prime} \mid s\right) \beta \frac{1}{\hat{\rho}_{c^{2}}\left(\theta_{-}, s^{\prime}\right)^{\sigma}}\right)^{-\frac{1}{\sigma}} \\
\hat{\rho}_{m^{2}}\left(\theta_{-}\right)=\left(\sum_{s^{\prime}=1}^{S} \pi\left(s^{\prime} \mid s\right) \beta \frac{1}{\left(e_{3}\left(s^{\prime}\right)-\theta_{-}\right)^{\sigma}}\right)^{-\frac{1}{\sigma}} .
\end{gathered}
$$

In the example below, it turns out that these functions can be extremely well approximated by low degree polynomials. Now one has to work with relative errors, since maximal relative consumption errors translate one-to-one to maximal relative errors in $\hat{\rho}_{m^{2}}$. With this, the equilibrium constraints of the maximization problem can be trivially written as

$$
\begin{array}{r}
-\tilde{q}(1+\epsilon) \hat{\rho}_{m^{1}}(\theta)+\left(e_{1}(s)-\tilde{q}^{\sigma} \theta\right)=0, \\
-\tilde{q}(1+\epsilon) \hat{\rho}_{m^{2}}(\theta)+\left(e_{2}(s)+\tilde{q}^{\sigma} \theta+\theta_{-}\right)=0 .
\end{array}
$$


Since the objective function now involves the ratio of actual consumption and approximate consumption policy, one has to verify, in addition to Steps 1-3 above, that the polynomial approximation $\hat{\rho}_{m^{1}}$ is sufficiently good, given the polynomial approximation for consumption.

To illustrate this, I consider a very simple numerical example. Suppose $S=2$, shocks are iid with $\pi=1 / 2$, endowments are given by

$$
e_{1}=\left(e_{1}(1), e_{1}(2)\right)=(1,2), \quad e_{2}=(4,2), \quad e_{3}=(0.5,0.5),
$$

and suppose $\beta=1$ and $\sigma=3$.

In the computed approximate equilibrium, it turns out that the endogenous state can be chosen to be $\hat{\Theta}=[-0.2,-0.05]$ and the approximate consumption functions can be chosen to be the quadratic functions $\hat{\rho}_{c^{1}}\left(\theta_{-}, 1\right)=1.6369+0.2350 \theta_{-}-0.0055 \theta_{-}^{2}$ and $\hat{\rho}_{c^{1}}\left(\theta_{-}, 2\right)=$ $1.2187+0.3227 \theta_{-}-0.0362 \theta_{-}^{2}$. With these specifications, a cubic polynomial leads to an excellent approximation for $\hat{\rho}_{m}$ and it turns out that for $a=1,2$

$$
\max _{\theta_{-} \in[-0.2,-0.05]}\left|\frac{\hat{\rho}_{m^{a}}\left(\theta_{-}\right)}{\left(\sum_{s^{\prime}=1}^{S} \pi\left(s^{\prime} \mid s\right) \beta \frac{1}{\hat{\rho}_{c^{a}}\left(\theta_{-}, s^{\prime}\right)^{\sigma}}\right)^{-\frac{1}{\sigma}}}-1\right|<10^{-8} .
$$

With this in place, one can now repeat the steps from above and verify that there is an exact equilibrium for which the approximation exhibits a relative error of less than

$10^{-4}$ : For $\delta=10^{-4}$, the optimal value of the maximization problem in Step 1 is around $4.7 \times 10^{-5}$, so even an additional error resulting from the approximation of $\hat{\rho}_{m}$ does not destroy robustness.

The simple example illustrates the method - the technique can easily handle examples with 6 or more states and risk aversion of 4 or 5 .

\section{Conclusion}

This paper develops a computationally feasible test to verify that a computed candidate equilibrium is close to a competitive equilibrium of a dynamic stochastic economy. The result has both practical and theoretical relevance.

In practice, researchers often want to argue that their computations of dynamic equilibria are accurate. Reporting relative errors in Euler-equations can certainly be useful for this, but only provides a necessary condition. The method in this paper can be used to give a bound on the exact deviation between computed function and actual equilibrium. The computation is relatively efficient and can be used for interesting small problems. I also argue that the method suggests an error analysis that is feasible for large-scale models.

Theoretically, it is known that the assumption of semi-algebraic preferences and technology allows for an arbitrarily good approximation of all equilibria of a finite economy (see e.g. Kubler and Schmedders (2010a)). The papers in the recent book edited by Brown and 
Kubler (2008) explore other implications of semi-algebraic fundamentals in finite economies. In this paper, I show how to extend the ideas from real algebraic geometry to infinite economies.

\section{Appendix}

I show that for the example in Section 2 there is no equilibrium close to the candidate Solution (2). The method from Kubler and Schmedders (2010b) proves that there is a unique steady state which is associated with candidate Solution (1). To understand better the dynamics of the model close to the approximate solution, it is useful to identify the pairs of beginning of period bond-holding and consumption of the middle aged, $\left(\theta_{-}, c\right)$, which lead to constant portfolios and those that lead to constant consumption. If portfolios are constant at some $\bar{\theta}=\theta=\theta_{-}$, the budget constraint and the first order condition of the middle aged imply as a necessary condition that there is a price $q$ such that $q \bar{\theta}=c-e_{2}-\bar{\theta}, \quad q c^{-3}=$ $\left(e_{3}-\theta\right)^{-3}$. Rewriting these equations as polynomials and using Gröbner bases to eliminate q (as in Kubler and Schmedders $(2010 \mathrm{~b})$ ), one finds that constant portfolios arise if $\left(\theta_{-}, c\right)$ satisfy the following polynomial equation.

$$
320 \theta_{-}^{4}+(-320 c+2904) \theta_{-}^{3}+(480 c-4836) \theta_{-}^{2}+\left(-320 c^{3}-240 c+2498\right) \theta_{-}+(40 c-423)=0 .
$$

If consumption remains constant, there must be a $\theta$ and a $q$ such that the budget constraint of the middle aged and both first order conditions hold, i.e.

$$
q \theta=c-e_{2}-\theta_{-}, \quad q c^{-3}=\left(e_{3}-\theta\right), \quad q\left(e_{1}+e_{2}+e_{3}-c-\left(e_{3}-\theta_{-}\right)\right)^{-3}=c^{-3} .
$$

Rewriting as polynomials and using Gröbner bases to eliminate $(q, \theta)$, one obtains

$$
\begin{array}{r}
256000 c^{4}+\left(-384000 \theta_{-}-4252800\right) c^{3}+\left(128000 \theta_{-}^{2}+2771200 \theta_{-}+14927120\right) c^{2}+ \\
\left(192000 \theta_{-}^{2}+4444800 \theta_{-}+25724280\right) c+ \\
\left(-64000 \theta_{-}^{3}-2222400 \theta_{-}^{2}-25724280 \theta_{-}-99252847\right)=0
\end{array}
$$

These two polynomial equations (9) and (10) define curves in $\left(\theta_{-}, c\right)$ space and one can easily determine the dynamics above and below these curves. Figure 2 shows the two curves, with $\theta_{-}$on the x-axis and $c$ on the y-axis in the region close to consumption of the second Candidate solution.

The arrows indicate the dynamics of $c$ and $\theta$. There are 4 relevant regions labeled 1-4. While it is not apparent from the figure, one can easily verify that the two curves do not intersect in this region. Therefore, there can be no equilibrium with consumption close to the one in candidate solution 2. Any such $\left(\theta_{-}, c\right)$ pair leads to a dynamical system that must leave Region 1 for either Region 2 or Region 3 and eventually must end up in Region 4 where consumption of the middle aged goes to infinity and therefore consumption of the old or of the young must become negative. This cannot be part of any competitive equilibrium. 


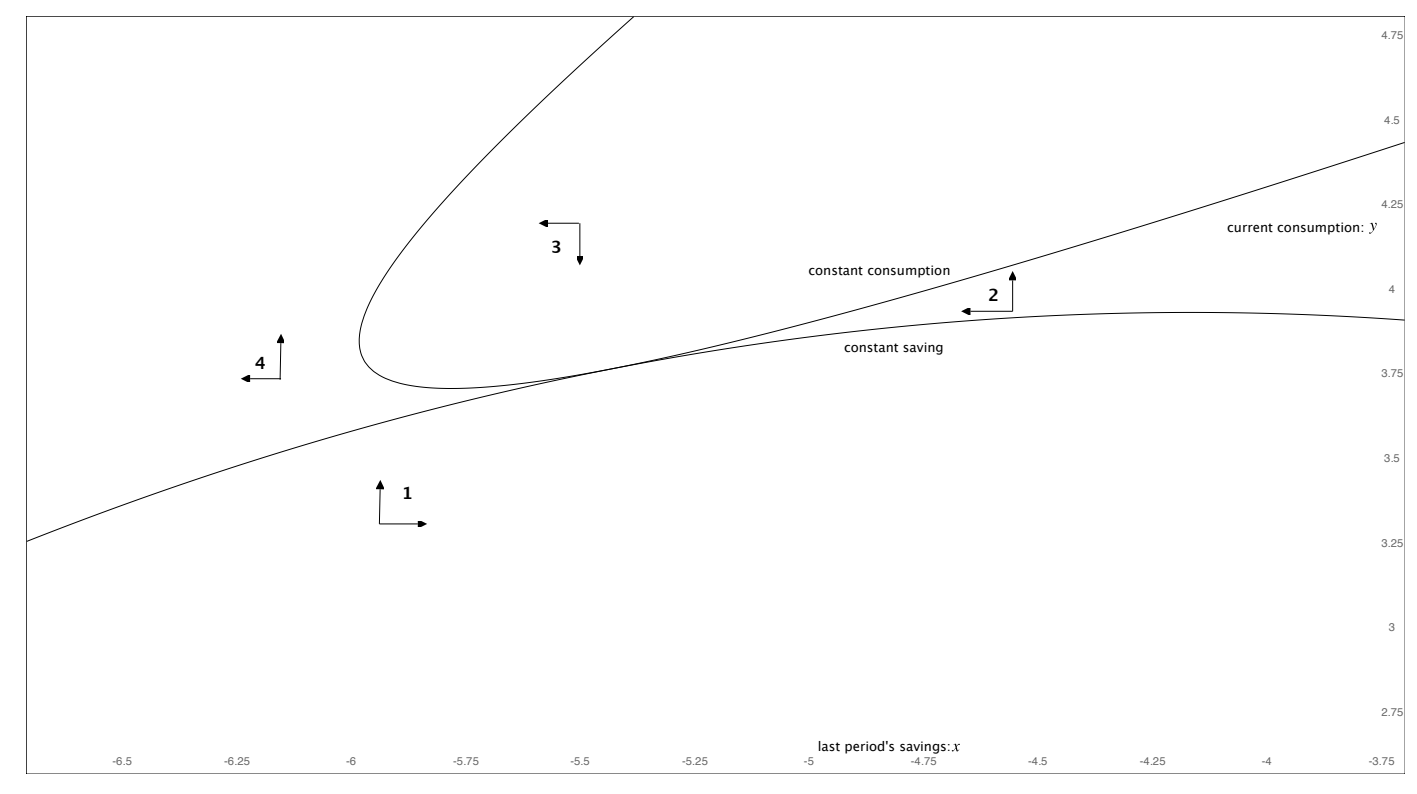

Figure 2

\section{References}

[1] Blume, L. and W.R. Zame, 1993, The algebraic geometry of competitive equilibrium, in W. Neuefeind, ed., Essays in General Equilibrium and International Trade: In Memoriam Trout Rader, Springer-Verlag, New York.

[2] Bochnak, J., M. Coste and M.-F. Roy, 1998, Real Algebraic Geometry, Springer-Verlag.

[3] Brown, D.J. and F. Kubler, 2008, Computational Aspects of General Equilibrium Theory, Springer-Verlag.

[4] Citanna, A. and P. Siconolfi, 2010, Existence of recursive equilibria in stochastic olg economies, Econometrica, 78, 309-347.

[5] Duffie, D., J. Geanakoplos, A. Mas-Colell, and A. McLennan, 1994, Stationary Markov Equilibria, Econometrica 62, 745-781.

[6] Judd, K.L., 1998, Numerical Methods in Economics, MIT Press.

[7] Kehoe, T.J. and D.K. Levine, 1990, The Economics of Indeterminacy in Overlapping Generations Models, Journal of Public Economics, 42, 219-243.

[8] Krueger, D. and F. Kubler, 2004, Computing Equilibrium in Stochastic OLG Models, Journal of Economic Dynamics and Control, 28, 1411-1436.

[9] Kubler, F. and H. Polemarchakis, 2004, Stationary Markov equilibria for overlapping generations, Economic Theory, 24, 623-643. 
[10] Kubler, F. and K. Schmedders, 2005, Approximate versus exact equilibria in dynamic economies, Econometrica, 73, 1205-1235.

[11] Kubler, F. and K. Schmedders, 2010a, Competitive Equilibria in Semi-Algebraic Economies, Journal of Economic Theory, 145, 301-330.

[12] Kubler, F. and K. Schmedders, 2010b, Uniqueness of steady states in overlapping generations, Journal of the European Economic Association, 8, 635-644.

[13] Laurent, M., 2009, Sums of squares, moment matrices and optimization over polynomials, in: M. Putinar and S. Sullivant, eds.: Emerging Applications of Algebraic Geometry, Springer Verlag.

[14] Luo, Z.-Q., J-.S. Pang and F. Ralph, 1996, Mathematical programs with equilibrium constraints, Cambridge University Press.

[15] Peyrl, H. and P.A. Parrilo, 2008, Computing sum of squares decompositions with rational coefficients, Theoretical Computer Science, 409, 269-281.

[16] Parrilo, P.A., 2003, Semidefinite programming relaxations for semialgebraic problems, Mathematical Programming, Ser. B, 96, 293-320.

[17] Samuelson, P., 1958, An exact consumption-loan model of interest with or without the social contrivance of money, The Journal of Political Economy, 66, 467-482.

[18] Santos, M.S., 2000, Accuracy of Numerical Solutions Using the Euler Equation Residuals, Econometrica 68, 1377-1402.

[19] Santos, M.S. and J. Vigo-Aguiar, 1998, Analysis of a Numerical Dynamic Programming Algorithm Applied to Economic Models, Econometrica 66, 409-426.

[20] Sturmfels, B., 2002, Solving Systems of Polynomial Equations, CBMS Regional Conference Series in Mathematics No. 97.

[21] Su, C.-L. and K.L. Judd, 2008, Constrained Optimization Approaches to Estimation of Structural Models, working paper, University of Chicago.

[22] Waki, H., S. Kim, M. Kojima and M. Muramatsu, 2006, Sums of Squares and Semidefinite Programming Relaxations for Polynomial Optimization Problems with Structured Sparsity, SIAM Journal on Optimization, 17, 218-242.

[23] Waki, H., S. Kim, M. Kojima and M. Muramatsu, 2008, SparsePOP : a Sparse Semidefinite Programming Relaxation of Polynomial Optimization Problems, ACM Transactions on Mathematical Software, 35, 15-23. 